\title{
Health Informatics Solutions in Response to COVID-19: Preliminary Insights from an International Survey
}

\author{
Seyedeh-Samin BARAKATI ${ }^{\mathrm{a}}$, Maxim TOPAZ ${ }^{\mathrm{b}}$, Laura-Maria PELTONEN ${ }^{\mathrm{c}}$, \\ James MITCHELL ${ }^{\mathrm{d}}$, Dari ALHUWAIL ${ }^{\mathrm{e}}$, \\ Tracie RISLING ${ }^{\mathrm{f}}$ and Charlene RONQUILLO ${ }^{\mathrm{a}, 1}$ \\ a Ryerson University \\ ${ }^{\mathrm{b}}$ Columbia University, New York, USA \\ ${ }^{\mathrm{c}}$ Department of Nursing Science, University of Turku, Finland \\ d School of Computing and Mathematics, Keele University, UK \\ ' Information Science Department, Kuwait University, Kuwait \\ ${ }^{f}$ University of Saskatchewan, Saskatoon, Canada
}

Keywords. medical informatics, COVID-19, pandemics, patient care technology

\section{Introduction}

Organizations and governments have prioritized the implementation of health information technologies (HIT) as a key tool in addressing the impacts of the COVID19 pandemic. With organizations shifting focus on high priority procedures, little opportunity is left for documentation and tracking the nature and scope of these technologies; much of this information is only known anecdotally. The aim of this ongoing study is to facilitate information sharing in the international health informatics community by collecting, synthesizing, and sharing information about the nature of HIT use in response to the COVID-19 pandemic.

\section{Methods}

This was a cross-sectional, exploratory, descriptive study that surveyed health informatics professionals internationally. A Web-based survey was developed using best practice guidance, in consultation with health informatics experts $[1 ; 2]$. The survey consists of 9 open-ended questions that cover the nature of HIT being used as part of the pandemic response. Ethical approval was obtained via Ryerson University Research Ethics Board. Convenience and snowball sampling were used. The sample comprised health informatics professionals who are involved with the development and deployment of HIT in healthcare settings. Thematic descriptive analyses of preliminary results were conducted to identify salient themes in the narrative survey responses [3]. In this paper, preliminary findings from data collected in May 2020

${ }^{1}$ Charlene Ronquillo, Daphne Cockwell School of Nursing, Ryerson University, 350 Victoria Street, Toronto ON, M5B 2K3, Canada; E-mail: cronquillo@ryerson.ca. 
are reported in response to the question: "What types of HIT are being used to address COVID-19 in the setting where you work?"

\section{Results and Discussion}

Fifty responses from eleven countries were analyzed. The majority of participants were health informaticians $(n=24)$, in IT related roles $(n=8)$ and management/decision making positions $(n=8)$. Three themes were identified in response to the types of HIT being used to address the COVID-19 pandemic. The first theme related to technologies for working remotely with patients and colleagues. For example, telehealth was reported to be deployed both within the care facility to minimize direct contact with patients and to connect with patients at home. The second theme pertained to technologies used in data collection, distribution, and analysis. Responses highlighted modifications made to electronic health record (EHR) systems to streamline data for reporting and pandemic planning. The third theme included technologies that were specifically developed to address COVID-19. This category included both expected (e.g. newly developed clinical decision support tools and standard terminology to be integrated into EHRs) and novel technologies (e.g. use of drones used for fever assessment). The types of HIT used by participants reflect necessary tools to respond to the challenges created by COVID-19 (e.g. remote working resulting from physical distancing requirements). The stated importance of data collection, management, and analytics tools solidify the power of data and the data-driven nature of healthcare as a key factor in planning and decision making in health care systems. The development of pandemic-specific solutions highlights the need to be nimble and innovative in order to mitigate the challenges posed by COVID19, although there is little overlap between the nature and use of HIT found in this study as compared to more visionary possibilities for HIT use as described elsewhere [4].

\section{Conclusion}

The preliminary results of this study provide insight into the diverse application of HIT for addressing COVID-19. This is consistent with the claims made by the World Health Organization which has identified HIT as "one of the most promising approaches to address this challenge in modern societies" [5]. Future research should advance the dialogue on HIT and pandemic planning with a focus on addressing concerns and creating clear actionable directives.

\section{References}

[1] Dillman DA, Smyth JD, and Christian LM. Internet, phone, mail, and mixed-mode surveys: the tailored design method, John Wiley \& Sons, 2014.

[2] Sue VM and Ritter LA. Conducting online surveys, SAGE publications, 2011.

[3] Vaismoradi M, Turunen H, and Bondas T. Content analysis and thematic analysis: Implications for conducting a qualitative descriptive study. Nursing \& Health Sciences 2013; 15: 398-405.

[4] Mahmood S, Hasan K, Colder Carras M, and Labrique A. Global Preparedness Against COVID-19: We Must Leverage the Power of Digital Health. JMIR Public Health Surveill 2020; 6, e18980.

[5] World Health Organization, Responding to community spread of COVID-19: interim guidance, 7 March 2020, in, World Health Organization, 2020. 